\title{
Review of: "Human Endometrial Organoids: Recent Research Progress and Potential Applications"
}

\author{
Yubao Wei
}

Potential competing interests: The author(s) declared that no potential competing interests exist.

The review paper wrote by Lou et al. give us a comprehensive perspectives of Endometrial Organoids per se. Endometrial Organoids is still in the infancy stage but has the potential to mimic the physiological or pathological status of the patient derived-tissues. The molecular mechanism of Endometrial stem cell reprograming is not illuminated, such as the role of wnt and notch signaling. So, the culture medium for Endometrial Organoids is contains dozens of supplements and it is not an efficient way. The key question is what is the Endometrial stem cell and how it is reprograming. Lou et al. presented many valuable information for the further study. 